\title{
Antenatal diagnosis of mucopolysaccharidosis type I (Hurler's disease) is not possible by electron microscopy of uncultured amniotic fluid cells*
}

\author{
GEORGE HUG, SHIRLEY SOUKUP, GAIL CHUCK, AND MARY RYAN \\ From the Division of Enzymology, Human Genetics, and Clinical Research Center, Children's Hospital \\ Medical Center, and the Department of Pediatrics, University of Cincinnati College of Medicine, Cincinnati, \\ Ohio 45229, USA.
}

SUMMARY Mucopolysaccharidosis type I (MPS I) is a lysosomal storage disorder characterised by the deficient activity of iduronidase and by the presence of MPS vacuoles in many tissues of affected patients. We studied whether these characteristics could be used for the antenatal diagnosis of the disease. We obtained amniotic fluid cells from two pregnancies at risk for MPS I, one pregnancy at risk for GSD II (another lysosomal disease), and eight normal control pregnancies. Measurements of iduronidase activity in cultured amniotic fluid cells indicated the presence of a MPS I fetus in one high risk pregnancy and an unaffected fetus in the other. This diagnosis was confirmed at delivery. On electron microscopy the uncultured amniotic fluid cells exhibited MPS-like vacuoles in the pregnancy with a GSD II fetus, in three of eight normal pregnancies, and in the pregnancy at risk for MPS I that had a normal fetus. No such vacuoles were seen in the pregnancy with the MPS I fetus. These false positive and false negative findings indicate that antenatal diagnosis of MPS I cannot be based on the electron microscopic presence or absence of MPS I vacuoles in uncultured amniotic fluid cells.

Mucopolysaccharidosis type I (MPS I, Hurler's disease) is a fatal lysosomal disorder of early childhood with autosomal recessive inheritance. MPS $I$ is characterised by deficient activity of lysosomal acid $\alpha$-L-iduronidase. On light and electron microscopy the cells of many tissues of MPS I patients, including those of liver and skin, exhibit MPS I vacuoles that are intracellular inclusions known as 'abnormal lysosomes'.'

The antenatal diagnosis of MPS I is possible by demonstrating the deficiency of iduronidase activity in cultured amniotic fluid cells. ${ }^{2}$ It has also been attempted by analysing such cells with the electron microscope for MPS I vacuoles. ${ }^{3}$ With these methods, the diagnosis is delayed by several weeks that are required for culturing the cells. In another lysosomal disease, glycogen storage disease type II (GSD II), that is characterised by the intracellular presence of GSD II vacuoles, this delay has been reduced to

*Supported by USPHS grant No RR00123-22 from the General Clinical Research Centers Branch, Division of Research Resources, National Institutes of Health.

Received for publication 5 January 1984.

Accepted for publication 13 February 1984. three days with the electron microscopic demonstration of GSD II vacuoles in uncultured amniotic fluid cells of affected pregnancies. ${ }^{4}$ In order to explore whether a similar use of the electron microscope in MPS I might result in a similar saving of time, we first examined the ultrastructure of uncultured amniotic cells from pregnancies at risk for MPS I. These ultrastructural observations were then compared with measurements of iduronidase activity in cultures derived from the cells of the same amniotic fluid specimens. We now report the comparative findings in these high risk pregnancies as well as in several pregnancies that served as controls.

\section{Patients and methods}

We studied two pregnancies at risk for MPS I (pregnancy 1 and pregnancy 2). These occurred in two unrelated families with previous MPS I children. The parents knew that they were obligatory carriers for the disease and requested antenatal diagnosis. Eight normal pregnancies were available for comparative studies, as was one pregnancy at risk for glycogen storage disease type II (GSD II) 


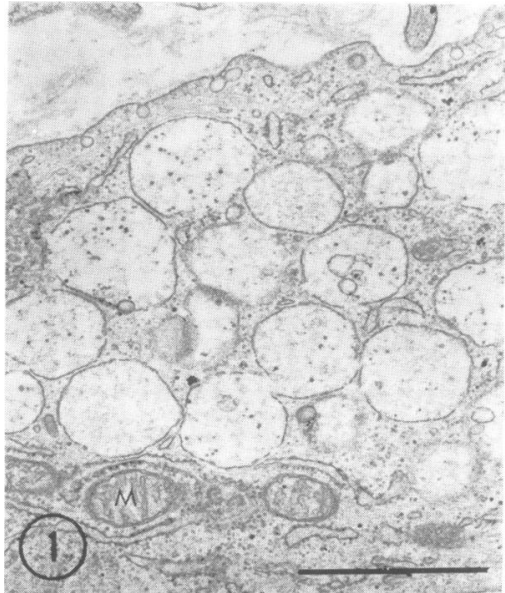

FIG 1 Electron photomicrograph of skin biopsy specimen from patient with MPS I. Although only a small part of one fibrocyte is visible, it contains more than a dozen MPS I vacuoles. Each vacuole is surrounded by a single membrane. It contains various cellular components such as ribosomes, glycogen particles, etc. For the most part, the vacuoles are nearly empty. $M=$ mitochondria. Bar $=2 \mu \mathrm{m}$.

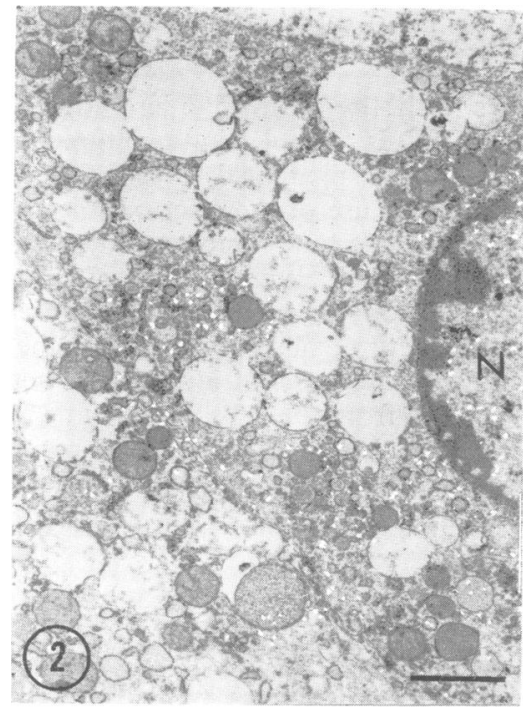

FIG 2 Electron photomicrograph of liver necropsy specimen of MPS I fetus at 19 weeks of gestation. MPS I vacuoles are ubiquitous and are visible within the hepatocyte. Each vacuole is surrounded by a single membrane. Most vacuoles are empty. $N=$ nucleus. $B a r=2 \mu m$. that subsequently was shown to have a GSD II fetus.

Amniocentesis was performed during the 15 th to 18th week of gestation. Cultures of amniotic fluid cells were established and were then analysed for $\overline{0}$ iduronidase activity. The simultaneous assay of 흠 other lysosomal enzyme activities showed the $\frac{\bar{\sigma}}{\bar{D}}$ viability of these cultures. Uncultured amniotic $\mathbb{\nabla}$ fluid cells of the same pregnancies were processed for electron microscopy. Thin sections of the plastic embedded cells were examined in a Phillips Electron Microscope for 2 to 4 hours. This examination was repeated at least twice. The target of the electron microscopic search was the MPS I vacuole. It is defined as an intracellular area separated from the rest of the cytoplasm by a single membrane. The membrane surrounded MPS I vacuole can be partially filled with fibrillar, floccular, or particulate material, membrane whorls, and cytoplasmic debris. These MPS I vacuoles have a circular appearance

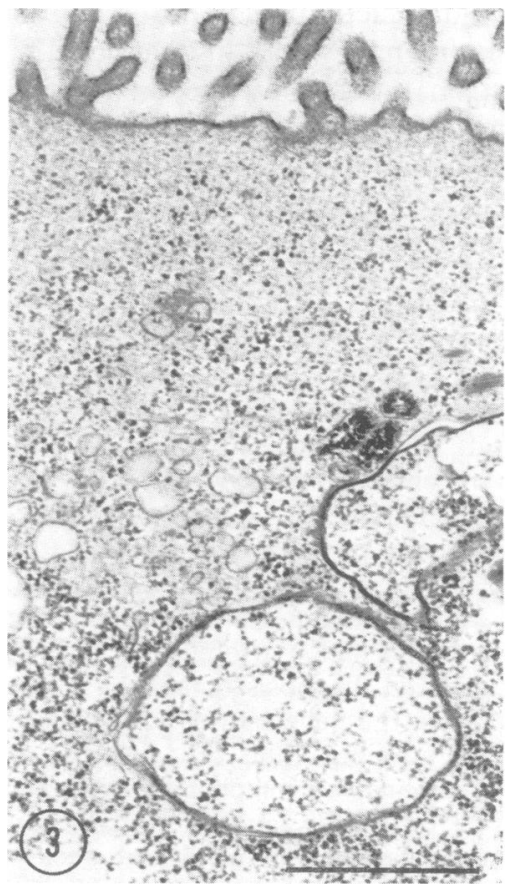

FIG 3 Electron photomicrograph of part of an uncultured amniotic fluid cell from pregnancy 2 at risk for MPS I but with a normal boy who was delivered at term. The pseudo MPS vacuole cannot be distinguished from the MPS I vacuoles depicted in figs 1 and 2 . The pseudovacuole contains cellular material, mostly $\beta$-glycogen particles. In this and other respects, the pseudovacuole is not different from the MPS I vacuoles seen in figs 1 and 2. Bar $=2 \mu \mathrm{m}$. 
TABLE Enzymatic analysis of cultured amniotic fluid cells.

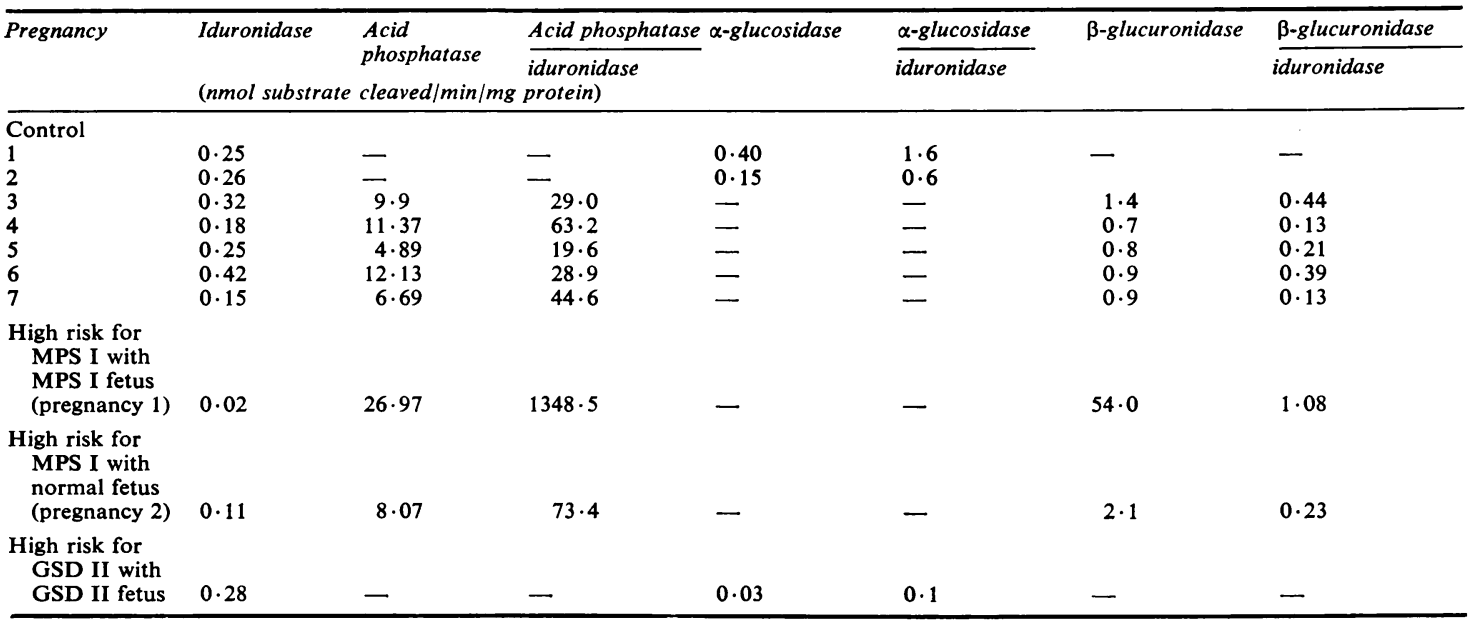

Different flasks of amniotic fluid cell cultures can have markedly different enzyme activities, probably because of differences in culture conditions that cannot be controlled. These differences tend to affect different enzyme activities of the same flask to a similar extent. This in vitro effect can thus be minimised by comparing in different flasks not the activity of the enzyme under study but the ratio of the activity under study and that of some marker enzyme. We divided the activity of the marker enzymes acid phosphatase, and/or $\alpha$-glucosidase, and/or $\beta$-glucuronidase by that of the study enzyme $\alpha$-L-iduronidase. These ratios are increased if the study enzyme is deficient. 'Control' signifies pregnancy with no known risk for MPS I. 'High risk for MPS I with MPS I fetus' signifies pregnancy 1 with MPS I fetus. 'High risk for MPS I with normal fetus' signifies pregnancy 2 at risk for MPS I that resulted in a normal boy. 'High risk for GSD II with GSD II fetus' signifies a pregnancy with a GSD II fetus.

and vary from 1 to $7 \mu \mathrm{m}$ in diameter with the majority measuring about $2 \mu \mathrm{m}$ across. These vacuoles are present in many tissues of MPS I patients, in particular in the cells of the liver and skin.

\section{Results}

In the affected older children of the two families, MPS I had been diagnosed by the characteristic clinical course and by the electron microscopic analysis of liver, skin fibroblast cultures, and other tissues when available. In the two affected children, a girl in family 1 and a boy in family 2 , the activity of iduronidase in liver and fibroblast cultures was not detectable. Electron microscopy of solid tissues exhibited the typical MPS I vacuoles (fig 1).

Results of the biochemical analysis of amniotic fluid cell cultures derived from control pregnancies and pregnancies 1 and 2 are listed in the table. The results indicated the presence of a MPS I fetus in pregnancy 1 and of an unaffected fetus in pregnancy 2. At delivery, this antenatal diagnosis was confirmed. In pregnancy 1 , fetal necropsy at 19 weeks' gestation showed iduronidase deficiency and MPS I vacuoles in the liver and other tissues (fig 2). In pregnancy 2 , a normal boy was delivered at term without the clinical, biochemical, or ultrastructural signs of MPS I.

Electron microscopic analysis of uncultured amniotic fluid cells from the affected high risk pregnancy 1 with a MPS I fetus did not indicate intracellular vesicles suggestive of MPS I vacuoles despite an extended search of 3 periods of several hours. Electron microscopic analysis of uncultured amniotic fluid cells from the unaffected high risk pregnancy 2 with a normal fetus indicated the

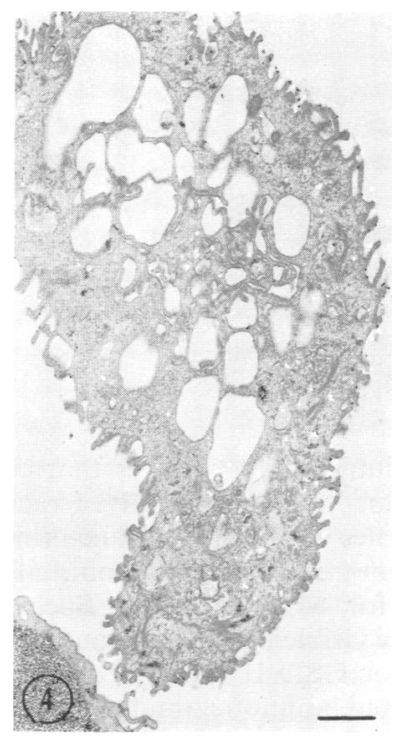

FIG 4 Electron photomicrograph of uncultured amniotic fluid cell from a pregnancy not at risk for MPS I. The pseudo MPS vacuoles within this cell cannot be distinguished from MPS I vacuoles present in figs 1 and 2. $B a r=2 \mu m$. 


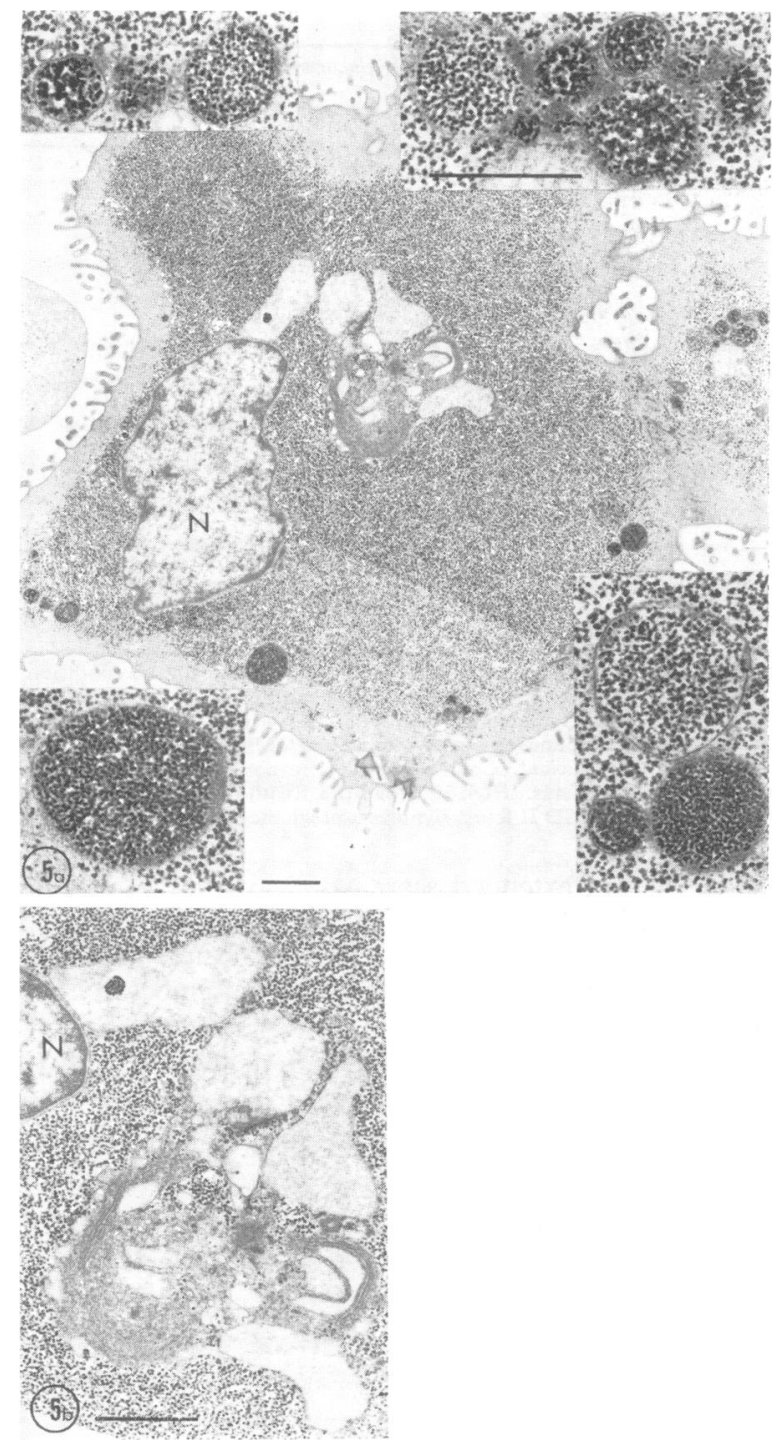

presence of intracellular vesicles that could not be differentiated from bona fide MPS I vacuoles (fig 3). Similar vacuoles were visible in uncultured amniotic fluid cells from three of the eight normal pregnancies not at risk for MPS I (fig 4). Such MPS I-like vacuoles were visible in one pregnancy at risk not for MPS I but for GSD II (fig 5). This pregnancy also had uncultured amniotic fluid cells with GSD II vacuoles and resulted in a GSD II fetus.

\section{Discussion}

The antenatal diagnosis of MPS I can be made by the
FIG 5 (a) Electron photomicrograph of an uncultured amniotic fluid cell from a pregnancy at risk for GSD II. The cell contains (non-diagnostic) cytoplasmic glycogen. In addition, this cell contains several GSD II vacuoles of which details can be seen in the insets. These GSD II vacuoles are surrounded by a single membrane and are completely filled with glycogen particles. These findings are diagnostic of a GSD II fetus and the diagnosis was confirmed by the delivery of a GSD II fetus. However, the same cell also contains several pseudo MPS vacuoles which cannot be distinguished from the MPS I vacuoles of figs 1 and 2. $N=$ nucleus, Bar $=1 \mu \mathrm{m}$. (b) Electron photomicrograph of a detail of the amniotic fluid cell depicted in (a) The picture is of four pseudo MPS vacuoles visible in the centre of $(a)$. Each vacuole is surrounded by a single membrane and contains cellular material resulting in an appearance that cannot be distinguished from the MPS I vacuoles visible in figs 1 and 2. Nevertheless, it is apparent that this cell of a GSD II fetus is not indicative of MPS I. The conclusion to be reached from this and the other figures is that the presence or absence of MPS-like vacuoles in amniotic? fluid cells cannot be used for either making or rejecting the diagnosis of MPS $I$ in the fetus. $N=$ nucleus. Bar= $I \mu m$.

demonstration of deficient iduronidase activity in cultures of amniotic fluid cells. Because of the time required for culturing these cells, the affected pregnancy may be more than 20 weeks old when the diagnosis is rendered. Electron microscopy of uncultured amniotic fluid cells can be completed within 3 days of the amniocentesis. Therefore, if the antenatal diagnosis of MPS could be made electron microscopically by examining these uncultured cells for the presence of MPS I vacuoles, the parents might retain some time dependent options. These options are important. MPS I is a slowly progressive disease. ${ }^{1}$ Although treatment of the disorder by bone marrow 
transplantation has recently been attempted, ${ }^{56}$ in many situations this would probably remain an untreatable disease even if the reported therapeutic success was confirmed.

An MPS I vacuole is an intracellular space surrounded by a single membrane and containing floccular, fibrillar, or membranous material, particulate matter including ribosomes, glycogen particles, and other cytoplasmic debris (fig 1). These vacuoles constitute 'abnormal lysosomes' that at times appear empty (fig 2). They are circular in appearance and have a diameter of 1 to $7 \mu \mathrm{m}$. MPS I vacuoles of this kind occur in solid tissues of patients with MPS I and in a variety of other lysosomal diseases. The latter include other types of mucopolysaccharidosis, generalised gangliosidosis, multiple sulphatase deficiency, and I cell disease. In general, these diseases cannot be treated effectively. The specific disease among these for which a given pregnancy is at risk will have been identified in the previous affected child by enzymatic tissue analysis. Therefore, the fact that similar MPS-like vacuoles occur in various lysosomal diseases does not preclude the use of the vacuoles for antenatal diagnosis, provided the vacuoles are present in uncultured amniotic fluid cells of affected pregnancies and are absent in such cells of unaffected pregnancies.

This, however, is not the case. We have observed MPS I vacuoles together with GSD II vacuoles in uncultured amniotic fluid cells of a pregnancy at risk for GSD II that subsequently produced a GSD II fetus (fig 5). We have observed MPS I vacuoles in uncultured amniotic fluid cells of normal pregnancies (fig 4). These false positive findings included one pregnancy at risk for MPS I that had a normal fetus by iduronidase activity in cultured amniotic fluid cells, by clinical presentation, and by biochemical and ultrastructural tissue analysis of a normal boy delivered at term (pregnancy 2, fig 3). These findings could reflect the heterozygous state for MPS I in the phenotypically normal infant. This hypothesis seems inconsistent with the fact that we also found MPS I vacuoles in three of eight normal control pregnancies not known to be at risk for lysosomal diseases including MPS I.

Conversely, we did not observe MPS I vacuoles in uncultured amniotic fluid cells of a high risk pregnancy that had a MPS I fetus, diagnosed by iduronidase deficiency in cultured amniotic fluid cells and by solid tissue analysis after delivery (pregnancy 1, fig 2). One might argue that this false negative finding could have been corrected by a further search for MPS I vacuoles in the uncultured amniotic fluid cells. This is not likely as the search had been extended to a total of 8 hours.
Our experience with the two pregnancies at risk for MPS I confirms the previous finding by others that antenatal diagnosis can be based on the measurements of iduronidase activity in cultured amniotic fluid cells.

Our experience indicates that electron microscopy of uncultured amniotic fluid cells must not be used for determining the presence or absence of mucopolysaccharidosis type $I$ in the fetus. We expect this to be correct for the other lysosomal disorders listed above that have intracellular vacuoles indistinguishable from those of MPS I. Attempts at saving time in diagnosing antenatally these disorders including MPS I might be successful if based on chorionic villus biopsy done early in the pregnancy? or on developing an oligonucleotide probe similar to the one available for $\alpha_{1}$-antitrypsin deficiency. ${ }^{8}$

At present the antenatal diagnosis by electron microscopy of uncultured amniotic fluid cells appears feasible only in glycogen storage disease type II. The GSD II fetus included in this study was so diagnosed in utero. ${ }^{9}$

\section{References}

1 McKusick VA, Neufeld EF. The mucopolysaccharide storage diseases. In: The metabolic basis of inherited disease. New York: McGraw-Hill, 1983:751-77.

${ }^{2}$ Hall CW, Liebaers I, Di Natale P, Neufeld EF. Enzyme diagnosis of the genetic mucopolysaccharide storage disorders. Methods Enzymol 1978;50:439-56.

${ }^{3}$ Ornoy A, Sekeles E, Cohen R, Kohn G. Electron microscopy of cultured skin fibroblasts and amniotic fluid cells in the diagnosis of hereditary storage diseases. Monogr Hum Genet 1978;10:32-9.

4 Hug G, Schubert WK, Soukup S. Prenatal diagnosis of type II glycogenosis. Lancet 1970;i:1002.

5 Hobbs JR, Barret AJ, Chambers D, et al. Reversal of clinical features of Hurler's disease and biochemical improvement after treatment by bone marrow transplantation. Lancet 1981 ;ii:709-12.

- Brady RO, Barranger JA, eds. The molecular basis of lysosomal storage disorders. New York: Academic Press, 1984.

7 Kolata G. First trimester prenatal diagnosis. a new method of prenatal diagnosis may largely replace amniocentesis. Science 1983;221:1031-2.

8 Kidd VJ, Tan ZK, Wallace BR, Itakura K, Woo SLC. Alpha ${ }_{1}$-antitrypsin deficiency and pulmonary emphysema: prenatal diagnosis by analysis of the chromosomal alpha ${ }_{1}$-antitrypsin gene. Pediatr Res 1983;17:7A.

- Hug G, Soukup S, Ryan M, Chuck G. Rapid prenatal diagnosis of glycogen-storage disease type II by electron microscopy of uncultured amniotic-fluid cells. $N$ Engl $J$ Med 1984;310:1018-22.

Correspondence and requests for reprints to Professor G Hug, Department of Pediatrics, Children's Hospital Medical Center, Elland and Bethesda Avenue, Cincinnati, Ohio 45229, USA. 